\title{
Antidepressant effects of aqueous extract of saffron and its effects on CREB, P-CREB, BDNF, and VGF proteins in rat cerebellum
}

\author{
Najmeh Asrari ${ }^{1}$, Rezvan Yazdian-Robati ${ }^{2}$, Khalil Abnous ${ }^{3,4}$, BiBi Marjan Razavi ${ }^{5}$, \\ Mrazieh Rashednia ${ }^{6}$, Faezeh Vahdati Hasani ${ }^{1}$ and Hossein Hosseinzadeh ${ }^{7 *}$ \\ ${ }^{1}$ School of Pharmacy, Mashhad University of Medical Sciences, Mashhad, Iran. \\ ${ }^{2}$ Department of Pharmaceutical Biotechnology, School of Pharmacy, Mashhad University of Medical Sciences, Mashhad, Iran. \\ ${ }^{3}$ Pharmaceutical Research Center, School of Pharmacy, Mashhad University of Medical Sciences, Mashhad, Iran. \\ ${ }^{4}$ Department of Medicinal Chemistry, School of Pharmacy, Mashhad University of Medical Sciences, Mashhad, Iran. \\ ${ }^{5}$ Targeted Drug Delivery Research Center, Department of Pharmacology and Toxicology, School of Pharmacy, Mashhad University of \\ Medical Sciences, Mashhad, Iran. \\ ${ }^{6}$ Department of Toxicology and pharmacology, School of Pharmacy, International branch, Shiraz University of Medical Sciences, Shiraz, Iran. \\ ${ }^{7 *}$ Corresponding author
}

\section{Key Words}

BDNF, CREB, Crocus sativus, Depression, P-CREB, VGF, Cerebellum

\begin{abstract}
Objective: The role of BDNF (brain-derived neurotrophic factor), CREB (cAMP response element binding) and VGF neuropeptide has been proved in antidepressant activity of long term saffron administration in the rat hippocampus. In this study we evaluated the role of these proteins in antidepressant activity of saffron in long term administration in the rat cerebellum.
\end{abstract}

Methods: Saffron aqueous extract (40 and $80 \mathrm{mg} / \mathrm{kg} /$ day) and imipramine (10 mg/kg/day) were administered intraperitoneally for 21 days to rats. At the end of experiment, animals were sacrificed and cerebellums were separated. The protein levels of BDNF, VGF, CREB and $\mathrm{P}$ - CREB in the rat cerebellum were evaluated using western blot analysis.

Received: May 10, 2017 Reviewed: Feb 14, 2018 Accepted: Feb 26, 2018

(c) This is an Open-Access article distributed under the terms of the Creative Common Attribution Non-Commercial License (http://creativecommons.org/licenses/by-nc/4.0/) which permits unrestricted noncommercial use, distribution, and reproduction in any medium, provided the original work is properly cited.

@ This paper meets the requirements of KS X ISO 9706, ISO 9706-1994 and ANSI/NISO Z39.48-1992 (Permanence of Paper).
Results: Saffron aqueous extract (80mg/kg/day) caused significant increase in protein level of P-CREB in long term treatment in the rat cerebellum. The increases in the protein levels of VGF, CREB and BDNF were not significant.

Conclusion: In summary, our results showed that antidepressant effect of saffron in rat cerebellum might be due to the enhanced phosphorylation of CREB.

\section{Introduction}

Depression is a mood disorder and the most common disabling problems of public health. Its prevalence in the world is around 15 to $20 \%$ [1]. It seems that this disorder is the result of complex interactions of genetic, epigenetic and environmental factors [2]. There is evidence that the cerebellum not only plays role in motor function and coordination of movement, but also contributes to an important role in emotion and cognition processing [3]. Result of quantitative meta-analytic study indicated that the cerebellum is one of the most recognized areas involved in the pathophysiology of depression [4]. Also Penga et al suggested that the 
cerebellum should be integrated in this illness [3]. Several biochemical and molecular pathways alter in response to antidepressant drugs, leading to alterations of gene expression. One candidate class of genes to be involved in the pathogenesis of mood disorders are neurotrophic factors, especially brain- derived neurotrophic factor (BDNF). BDNF is a small dimmer neuroprotective protein involved in maintenance and cell survival of nervous system. This protein is regulated by CREB (cAMP response element binding protein). It has been reported in depressive condition, BDNF levels in the brain is reduced [5]. CREB is a transcription factor that known as molecular convergence point of antidepressant treatment and involved in the regulation of neurogenesis [6]. CREB is activated by phosphorylation at specific serine residue and also up regulated by chronic antidepressants treatment $[7,8]$. Another gene contributed in depressive mechanism is VGF. VGF is a neuropeptides which its transcription dependent on CREB level and up regulated in antidepressant treatment [9]. Due to safety concerns and side effects of many antidepressant drug including anxiety and loss of appetites, herbal medicine as alternatives and safe medications is essential for the treatment of major depressive disorder (MDD) $[6,10]$. Saffron is one of the promising herbal compounds for the cure of depression based on results from recent clinical trials study $[11,12]$

Crocus sativus L., known as saffron, is a perennial herbaceous plant has long been used in traditional and modern medicines as an antioxidant [13], antidepressant [14], anti-tumor [15,16], anticonvulsive [17-19], improvement of spatial cognitive and memory impairements and morphine morphine withdrawal syndrome [20-22], antinociceptive [23, 24], antisolar [25, 26], cardioprotective [27-29], hepatoprotective [30] and neuroprotective [31, 32]. Moreover, protective effects of saffron against natural or chemical toxicities and in metabolic syndrome have been proved in several studies [33, 34]. In addition, saffron offers promise for the treatment of depressive disorder [35]. The neuroprotective and antidepressant effects of saffron and crocin (constituent of saffron) in rats exposed to the forced swimming test were studied and results showed crocin dose-dependently enhanced levels of CREB and BDNF, and, at higher levels, increased VGF in hippocampus [36, 37]. Also the aqueous extract of saffron elevated protein expressions of BDNF, CREB, P-CREB and transcription levels of BDNF in the hippocampus of rat [37]. In the present study, the protein levels of CREB, BDNF, and VGF in rat cerebellum were measured to understand the underlying molecular mechanism of antidepressant effects of aqueous extract of saffron in cerebellum.

\section{Materials and Methods}

\subsection{Plant extract}

C. sativus stigma from Novin Saffron Co. was collected from Ghaen, Khorasan province, Northeast of Iran. A voucher specimen (No: 11135) has been deposited in the Herbarium of School of Pharmacy, Mashhad University of Medical Sciences, Mashhad, Iran. To prepare the aqueous extract of C. sativus $8 \mathrm{~g}$ of stigma powder was macerated in $300 \mathrm{~mL}$ distilled water for $72 \mathrm{~h}$ in the refrigerator while shaking. Following centrifuging, supernatant was freezedried for $24 \mathrm{~h}$.

\subsection{Chemicals}

Imipramine hydrochloride bought from Marham Daru, Iran.Tris-HCl, (Sodium fluoride $(\mathrm{NaF})$, sodium orthovanadate, EDTA, complete protease inhibitor cocktail (P8340), Sodium dodecyl sulfate (SDS), 2-mercaptoethanol (2-ME), glycerol, Tween 20 and phenylmethylsulfonyl fluoride (PMSF), purchased from Sigma- Aldrich, Germany.

\subsection{Animals}

Adult male Wistar Albino rats, weighing 250-300 g, were obtained by the Animal Center of MUMS. 24 rats were housed in standard plastic cages in the colony room under 12-h light/dark cycle, $22 \pm 2{ }^{\circ} \mathrm{C}$ and $40-50 \%$ humidity conditions with free access to food and water before and during the study. All the experiments in this study were performed according to Mashhad University of Medical Sciences, Ethical Committee Acts. Rats were divided in four groups $(n=6)$. Animals in control group were injected with saline and treated group injected (i.p.) with aqueous extract of saffron (40 and $80 \mathrm{mg} / \mathrm{kg}$ ) and imipramine (10 $\mathrm{mg} / \mathrm{kg} /$ day) group, as positive control. Treatments were continued for 21 days. After 21 days rats were sacrificed and cerebellums were removed and dissected on ice immediately, rinsed and then frozen in liquid nitrogen and stored at $-80^{\circ} \mathrm{C}$ until further analyses.

\subsection{Protein extraction}

In order to prepare samples for western blotting, 200 $\mathrm{mg}$ of frozen cerebellums were lysed in the homogenization buffer containing )Tris- $\mathrm{HCl} 50 \mathrm{mM}$ ( $\mathrm{pH}$ : 7.4), 2 mM EDTA, 10 mMNaF, 1 mM Na3VO4, 10 mM $\beta$-glycerol phosphate, $0.2 \% \mathrm{w} / \mathrm{v}$ NaDC, $1 \mathrm{mM}$ PMSF, and complete protease inhibitor cocktail ( using polytron homogenizer (POLYTRON ${ }^{\circ}$ PT 10-35, Kinematica, Switzerland) on ice. Samples incubated for $2 \mathrm{~h}$ at $4^{\circ} \mathrm{C}$ then centrifuged at 10000 $\times \mathrm{g}$ for $15 \mathrm{~min}$ at $4^{\circ} \mathrm{C}$ and then supernatants were collected and protein contents were determined by Bio-Rad Protein Assay Kit.

\subsection{Western blot}

Western blot analysis carried out on the prepared samples to assess the levels of CREB, P-CREB, BDNF and VGF. In brief, Equal volumes of supernatants were mixed with SDS sample buffer containing 100 mMTris-base, $4 \% \mathrm{w} / \mathrm{v}$ SDS, $20 \% \mathrm{v} / \mathrm{v}$ glycerol, $10 \% \mathrm{v} / \mathrm{v} 2$-mercaptoethanol and $0.2 \%$ $\mathrm{w} / \mathrm{v}$ bromophenol blue and incubated in boiling water for $5 \mathrm{~min} .10 \mathrm{mg} / \mathrm{mL}$ from each samples were loaded on SDSPAGE then transferred to PVDF (polyvinylidenedifluoride) membrane following electrophoresis. Membranes were blocked with 5\% skim milk in TBST for $3 \mathrm{~h}$ at room temperature. Blots were probed with specific primary antibodiy, mouse monoclonal anti-serum against P-CREB 
(Ser133) (\#9196, Cell Signaling, USA), rabbit monoclonal anti- serum against CREB (\#9197, Cell Signaling, USA),rabbit polyclonal antiserum against BDNF (\#ab46176, Abcam, USA) and VGF(\#ab74140, Abcam, USA), at 1:1000 dilutions for $2 \mathrm{~h}$ at room temperature. Membranes were washed 3 times with TBST. Then, blots were incubated with anti-mouse and rabbit horse radish peroxidase labeled IgG(\#7076 and \#7074, Cell Signaling, USA) as secondary antibody at 1:3000 dilutions for $1 \mathrm{~h}$ at room temperature while shaking. Finally, protein bands were visualized by an enhanced chemiluminescence reagent (Pierce ECL western blotting substrate) and Alliance Gel-doc (Alliance 4.7 Gel doc, UVtec UK). UV Tec software (UK) was used to analyse protein bands intensities. All protein bands were normalized against intensities of corresponding $\beta$-actin (\# 3700 and \# 4970, Cell Signaling, USA).

\subsection{Statistical analysis}

For analyzing, we used Graph Pad InStat version 5.00 (Graph Pad Software, San Diego, California, USA) with One-way Analysis of Variance (ANOVA) followed by Tukey post-hoc test and plotted in Graph Pad Prism version 5.00 (Graph Pad Software, San Diego California USA). All data offered as mean \pm Standard error of the mean (S.E.M). P values less than 0.05 were regard as statistical significance.

\section{Results}

In this project, following previous our study (22), we used two dose of aqueous extract of saffron $(40 \mathrm{mg} / \mathrm{kg}$ and 80 $\mathrm{mg} / \mathrm{kg}$ ). Effects of the subacute treatment with aqueous extract of saffron $(40 \mathrm{mg} / \mathrm{kg}$ and $80 \mathrm{mg} / \mathrm{kg}$, IP) on the p-CREB, CREB, BDNF, and VGF protein expression in the cerebellum are illustrated.

\subsection{Effect of aqueous extract of saffron on the protei level of BDNF in rat cerebellum}

The aqueous extracts of saffron showed no noticeable effect on protein levels of BDNF compare with saline group (Fig. 1).

\subsection{Effect of aqueous extract of saffron on the protein levelof VGF in rat cerebellum}

According to Fig. 2. the aqueous extracts of saffron could not change the protein level of VGF in rat cerebellum.

\subsection{Effect of aqueous extract of saffron on the protein levels of CREB in rat cerebellum}

Based on our result illustrated in Fig. 3, the aqueous extracts of saffron could not change the protein level of CREB in rat cerebellum.

\subsection{Effect of aqueous extract of saffron on the protein levels of $\mathrm{p}$-CREB in rat cerebellum}

The aqueous extract of saffron ) $80 \mathrm{mg} / \mathrm{kg}$ ( increased the levels of $\mathrm{p}$-CREB, compared with normal saline group $(\mathrm{P}<0.001)$ (Fig. 4).

\section{Discussion}

In this project, the effects of subacute administration of aqueous extract of saffron on protein levels of P-CREB, CREB, BDNF, and VGF, in rat cerebellum were evaluated by western blotting. It has been reported as a result of neuronal atrophy, stress and reduced neurogenesis, depression may occur that lead to up-regulation of CREB protein and other neurotrophic factors, mainly BDNF which its transcription depends on CREB. BDNF also regulates VGF gene [38]. According our data, the protein levels of BDNF, CREB and VGF, at both doses of aqueous extract were not significantly changed. However, the aqueous extract of saffron $(80 \mathrm{mg} / \mathrm{kg}$ ) increased the protein level of P-CREB. Chronic administration of different antidepressant drugs, promote the expression of CREB mRNA in rat hippocampus and CREB protein are enhanced along with its mRNA indicating a role for the cAMP cascade [7]. In two separated studies crocin and aqueous extract of saffron increased P-CREB level in rat hippocampus [36, 37]. The level of P-CREB was upregulated by desmethylimipramine and fluoxetine in other regions of the brain [39]. Some antidepressants have shown different effects on CREB protein. Laifenfeld et al reported administration of desipramine and fluoxetine for 21 days could increase the protein level of P-CREB, active form of CREB, in the frontal cortex [40]. In addition to hippocampus, cerebellum has an important role in control of emotion and cognitive processing besides its role in motor coordination [3]. So that, white matter microstructural abnormalities in depressed patients have been observed in some brain areas including the cerebellum [41]. Our finding from this study indicated that chronic administration of saffron aqueous extract may lead to the up regulation of P-CREB in rat cerebellum.

\section{Conclusion}

It is concluded that the mechanism of aqueous extract of saffron as an antidepressant, is specially mediated by increasing the P-CREB protein expressions in rat cerebellum.

\section{Acknowledgments}

The authors are thankful to the Vice Chancellor of Research, Mashhad University of Medical Sciences for financial support. The results described in this paper are part of a Pharm. D. thesis of Najmeh Asrari.

\section{Conflict of interest}

The authors have declared no conflict of interest. 


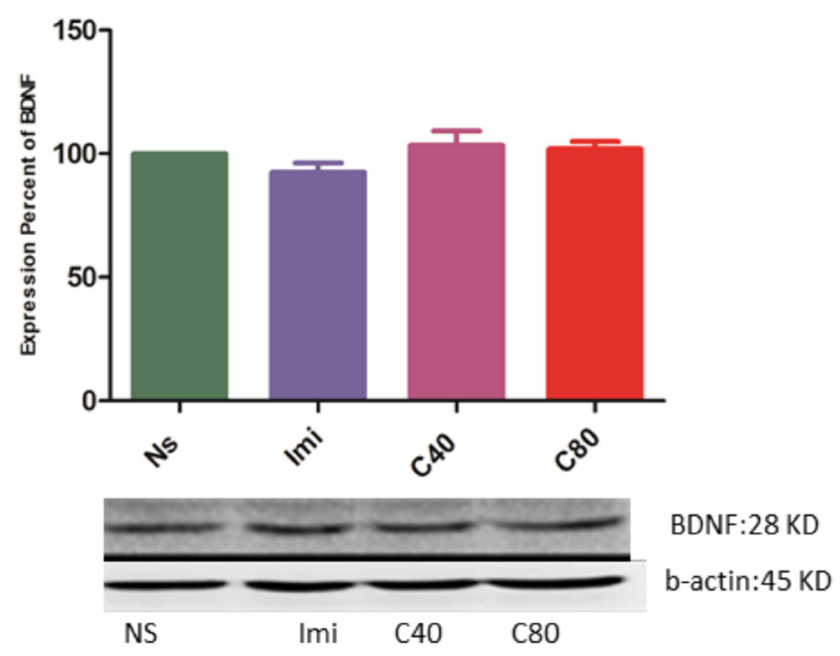

Figurel Effect of aqueous extract of saffron on the BDNF level in the rat cerebellum by western blotting (NS = Normal Saline, $\mathrm{c}=$ Saffron, $\mathrm{IMi}=$ Imipramine). a: Representative western blots showing specific bands for BDNF and $\bigotimes$-actin as an internal control. These bands are representative of 6 separate experiments. $b$ : Densitometric data of protein analysis. Data are expressed as the mean \pm SEM. Statistical analysis was performed using one-way ANOVA

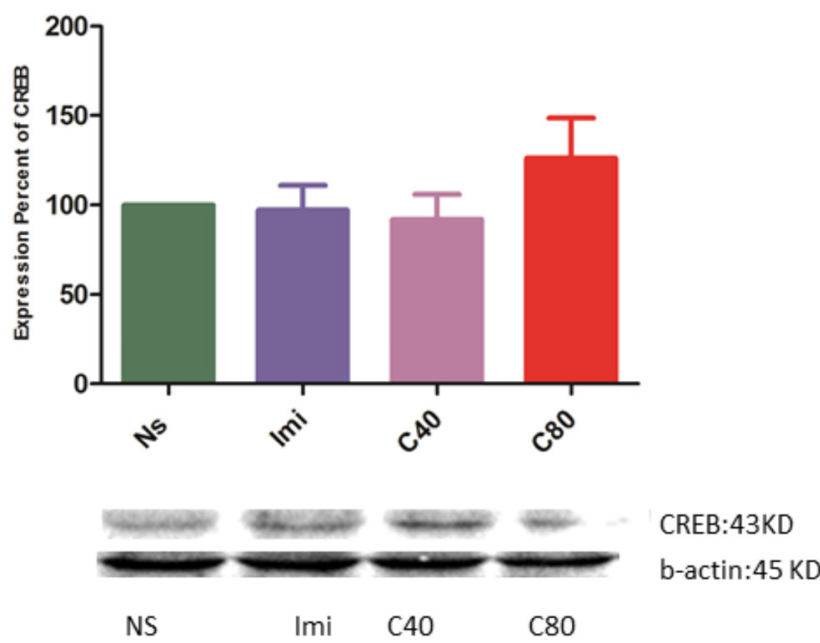

Figure 3 Effect of aqueous extract of saffron on the CREB level in the rat cerebellum by western blotting (NS = Normal Saline, $\mathrm{c}=$ Saffron, $\mathrm{IMi}=$ Imipramine $)$. a:Representative western blots showing specific bands for BDNF and $\bigotimes$ - actin as an internal control. These bands are representative of 6 separate experiments. $\mathrm{b}$ :Densitometric data of protein analysis.Data are expressed as the mean \pm SEM. Statistical analysis was performed using oneway ANOVA

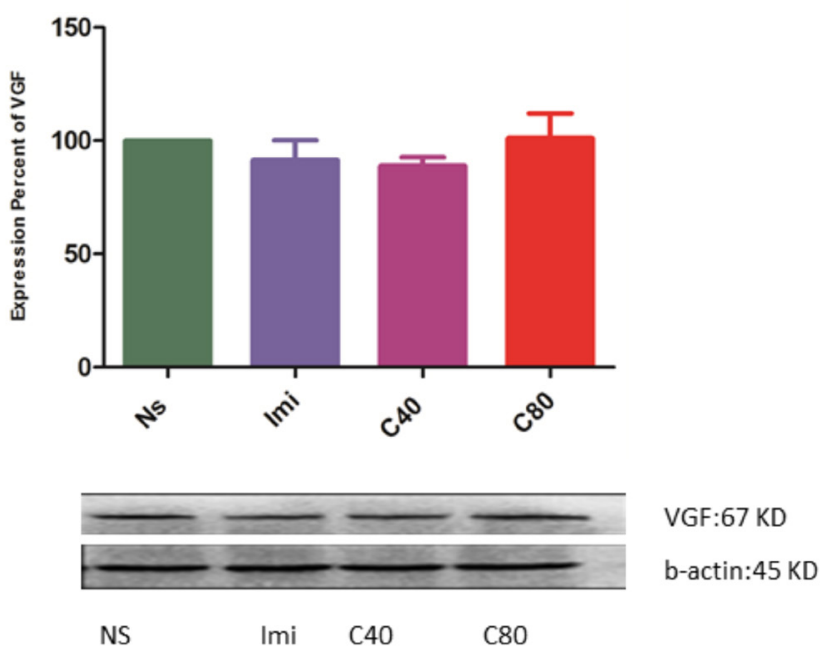

Figure 2 Effect of aqueous extract of saffron on the VGF level in the Rat cerebellum by western blotting (NS = Normal Saline, $\mathrm{c}=$ Saffron, $\mathrm{IMi}=$ Imipramine). a: Representative western blots showing specific bands for BDNF and $\bigotimes$ - actin as an internal control. These bands are representative of 6 separate experiments. b: Densitometric data of protein analysis.Data are expressed as the mean \pm SEM. Statistical analysis was performed using one-way ANOVA

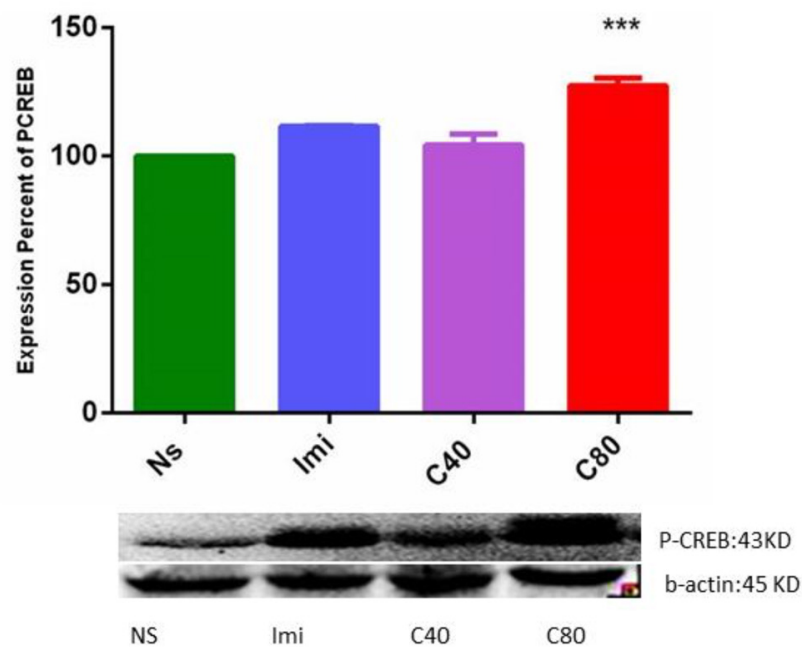

Figure 4 Effect of aqueous extract of saffron on the P-CREB level in the Rat cerebellum by western blotting (NS = Normal Saline, $\mathrm{c}=$ Saffron, Imi = Imipramine). a:Representative western blots

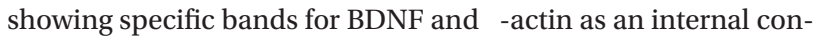
trol. These bands are representative of 6 separate experiments. $\mathrm{b}$ :Densitometric data of protein analysis.Data are expressed as the mean \pm SEM. Statistical analysis was performed using oneway ANOVA followed by Tukey-Kramer post test for multiple comparisons. ${ }^{* * *} \mathrm{P}<0.001$ compared to the normal saline group. 


\section{References}

1. Berton O, Nestler EJ. New approaches to antidepressant drug discovery: beyond monoamines. Nature Rev Neurosci. 2006;7(2):137-51.

2. Haenisch B, Bonisch H. Depression and antidepressants: insights from knockout of dopamine, serotonin or noradrenaline re-uptake transporters. Pharmacol Ther. 2011;129(3):352-68.

3. Penga J, Liua J, Nieb B, Li Y, Shanb B, Wangc G, et al. Cerebral and cerebellar gray matter reduction in first-episode patients with major depressive disorder: A voxel-based morphometry study. Eur J Radiol. 2011;80: 395-9.

4. Zeng L, Liu L, Liu Y, Shen H, Li Y, Hu D. Antidepressant Treatment Normalizes White Matter Volume in Patients with Major Depression. Plos One. 2012;7(8): e44248.

5. Yulug B, Ozan E, Gönül AS, Kilic E. Brain-derived neurotrophic factor, stress and depression: a minireview. Brain Res Bull. 2009;78(6):267-9.

6. Sarris J, Panossian A, Schweitzer I, Stough C, Scholey A. Herbal medicine for depression, anxiety and insomnia: a review of psychopharmacology and clinical evidence. Eur Neuropsychopharmacol. 2011;21(12):841-60.

7. Nibuya M, Nestler EJ, Duman RS. Chronic antidepressant administration increases the expression of cAMP response element binding protein (CREB) in rat hippocampus. J Neurosci. 1996;16(7):2365-72.

8. Gass P, Riva MA. CREB, neurogenesis and depression. BioEssays. 2007;29(10):957-61.

9. Thakker-Varia S, Alder J. Neuropeptides in depression: role of VGF. Behav Brain Res. 2009;197(2):262-78.

10. Hosseinzadeh H, Nassiri冈Asl M. Avicenna's (Ibn Sina) the canon of medicine and saffron (Crocus sativus): a review. Phytother Res. 2013;27(4):475-83.

11. Mollazadeh H, Emami SA, Hosseinzadeh H. Razi's Al-Hawi and saffron (Crocus sativus): a review. Iran J Basic Med Sci. 2015;18(12):1153-6.

12. Moshiri M, Vahabzadeh M, Hosseinzadeh H. Clinical applications of saffron (Crocus sativus) and its constituents: a review. Drug Res. 2015;65(6):287-95.

13. Hosseinzadeh H, Shamsaie F, Mehri S. Antioxidant activity of aqueous and ethanolic extracts of Crocus sativus L. stigma and its bioactive constituents, crocin and safranal. Pharmacogn Mag. 2009;5(20):419-24.

14. Hosseinzadeh H, Karimi G, Niapoor M. Antidepressant effect of Crocus sativus L. stigma extracts and their constituents, crocin and safranal, in mice. Acta Hort. 2004;650: 435-445.

15. Behravan J, Hosseinzadeh H, Rastgoo A, Mohamad ali pour malekashah O. Hessani M. Evaluation of the cytotoxic activity of crocin and safranal using potato disc and brine shrimp assays. Physiol Pharmacol. 2010;13(4):397-403.

16. Rastgoo M, Hosseinzadeh $\mathrm{H}$, Alavizadeh $\mathrm{H}$, Abbasi A, Ayati Z, Jaafari MR.

Antitumor activity of PEGylated nanoliposomes containing crocin in mice bearing C26 colon carcinoma. Planta Med. 2013;79(6): 447-451.

17. Hosseinzadeh H, Sadeghnia, H.R. Protective effect of safranal on pentylenetetrazol-induced seizures in the rat: Involvement of GABAergic and opioids systems. Phytomedicine. 2007;14(4): 256-262.

18. Hosseinzadeh $\mathrm{H}$, Talebzadeh F. Anticonvulsant evaluation of safranal and crocin from Crocus sativus in mice. Fitoterapia. 2005;76(7-8): 722-724.

19. Sadeghnia HR, Cortez MA, Liu D, Hosseinzadeh H, Snead CO. Antiabsence effects of safranal in acute experimental seizure models: EEG and autoradiography. J Pharm Pharm Sci. 2008;11(3):1-14.

20. Hosseinzadeh H, Ziaei T. Effects of Crocus sativus stigma extract and its constituents, crocin and safranal, on intact memory and scopolamine-induced learning deficits in rats performing the Morris water maze task. J Med Plants. 2006; 5 (19): 40-50.

21. Hosseinzadeh H, Sadeghnia HR, Ghaeni FA, Motamedshariaty VS, Mohajeri SA. Effects of saffron (Crocus sativus L.) and its active constituent, crocin, on recognition and spatial memory after chronic cerebral hypoperfusion in rats. Phytother Res. 2012;26(3):381-6.

22. Hosseinzadeh, H., Jahanian, Z. Effect of Crocus sativus L. (saffron) stigma and its constituents, crocin and safranal, on morphine withdrawal syndrome in mice. Phytother Res. 2010; 24 (5):726-730

23. Hosseinzadeh H, Shariaty VM. Anti-nociceptive effect of safranal, a constituent of Crocus sativus (saffron), in mice. Pharmacologyonline, 2007; 2: 498-503.

24. Amin B., Hosseinzadeh H. Evaluation of aqueous and ethanolic extracts of saffron, Crocus sativus L., and its constituents, safranal and crocin in allodynia and hyperalgesia induced by chronic constriction injury model of neuropathic pain in rats. Fitoterapia. 2012;83(5): 888-895.

25. Golmohammadzadeh S, Jaafari MR, Hosseinzadeh H. Does saffron have antisolar and moisturizing effects? Iran J Pharm Res. 2010;9(2):133-140.

26. Golmohammadzadeh S, Imani F, Hosseinzadeh $\mathrm{H}$, Jaafari MR. Preparation, characterization and evaluation of sun protective and moisturizing effects of nanoliposomes containing safranal. Iran J Basic Med Sci. 2011;14(6): 521-533.

27. Mehdizadeh R, Parizadeh MR, Khooei AR, Mehri S, Hosseinzadeh H. Cardioprotective effect of saffron extract and safranal in isoproterenol-induced myocardial infarction in wistar rats. IJBMS. 2013;16(1):56-63.

28. Razavi BM, Hosseinzadeh H, Movassaghi AR, Imenshahidi M, Abnous K. Protective effect of crocin on diazinon induced cardiotoxicity in rats in subchronic exposure.Chem-Bio Interact. 2013a 203 (3): 547-555.

29. Razavi M, Hosseinzadeh H, Abnous K, Motamedshariaty VS, Imenshahidi M. Crocin restores hypotensive effect of subchronic administration of diazinon in rats. Iran J Basic Med Sci. 2013b;16(1): 64-72.

30. Lari P, Abnous K, Imenshahidi M, Rashedinia M, Razavi $\mathrm{M}$, Hosseinzadeh $\mathrm{H}$. Evaluation of diazinon-induced hepatotoxicity and protective effects of crocin. Toxicol Indust

Health, 2015;31(4): 367-376.

31. Nassiri-Asl M, Hosseinzadeh H. Neuropharmacology Effects of Saffron (Crocus sativus) and Its Active Constituents. Bioactive Nutraceuticals and Dietary Supple- 
ments in Neurological and Brain Disease. Prevention and Therapy, 2015;29.39

32. Dorri SA, Hosseinzadeh H, Abnous K, Hasani FV, Robati RY, Razavi BM. Involvement of brain-derived neurotrophic factor (BDNF) on malathion induced depressive-like behavior in subacute exposure and protective effects of crocin. Iran J Basic Med Sci, 2015;18(10): 958-86.

33. 33. Razavi BM, Hosseinzadeh H. Saffron as an antidote or a protective agent against natural and chemical toxicities. DARU, J Pharm Sci. 2015;23(1): 112.

34. Razavi BM, Hosseinzadeh H. Saffron: a promising natural medicine in the treatment of metabolic syndrome. J Sci Food Agric. 2017;97(6):1679-1685.

35. Akhondzadeh S, Fallah-Pour H, Afkham K, Jamshidi AH, Khalighi-Cigaroudi F. Comparison of Crocus sativus L. and imipramine in the treatment of mild to moderate depression: a pilot double-blind randomized trial. BMC Complement Altern Med. 2004;4(1):12.

36. Vahdati Hassani F, Naseri V, Razavi BM, Mehri S, Abnous $\mathrm{K}$, Hosseinzadeh $\mathrm{H}$. Antidepressant effects of crocin and its effects on transcript and protein levels of CREB, BDNF, and VGF in rat hippocampus. DARU, J Pharm Sci. 2014; 22(1):16.

37. Ghasemi T, Abnous K, Vahdati F, Mehri S, Razavi B, Hosseinzadeh H. Antidepressant effect of Crocus sativus aqueous extract and its effect on CREB, BDNF, and VGF transcript and protein levels in Rat hippocampus. Drug Res (Stuttq). 2015;65(7):337-43.

38. Aydemir O, Deveci A, Taneli F. The effect of chronic antidepressant treatment on serum brain-derived neurotrophic factor levels in depressed patients: a preliminary study. Prog Neuropsychopharmacol Biol Psychiatry. 2005;29(2):261-5.

39. Blendy JA. The Role of CREB in Depression and Antidepressant Treatment. Biol Psychiatry. 2006;59(12):1144-50.

40. Laifenfeld D, Karry R, Grauer E, Klein E, Ben-Shachar D. Antidepressants and prolonged stress in rats modulate CAM-Ll, laminin, and pCREB, implicated in neuronal plasticity. Neurobiol Dis. 2005;20(2):432-41.

41. Alexopoulos GS, Murphy CF, Gunning-Dixon FM, Latoussakis V, Kanellopoulos D, Klimstra S, et al. Microstructural white matter abnormalities and remission of geriatric depression. Am J Psychiatry. 2008;165(2):238-44. 